\title{
Obscured quasars: the link between star-formation and black hole activity
}

\author{
Vincenzo Mainieri $^{1}$, Angela Bongiorno ${ }^{2}$ \\ and the COSMOS team \\ ${ }^{1}$ ESO, Karl-Schwarschild-Strasse 2, D-85748 Garching bei München, Germany \\ email: vmainier@eso.org \\ ${ }^{2}$ INAF-Osservatorio Astronomico di Roma, via Frascati 33, 00040 Monteporzio-Catone, Italy
}

\begin{abstract}
We explore the connection between black hole growth at the center of obscured quasars selected from the XMM-COSMOS survey and the physical properties of their host galaxies. We study a bolometric regime $\left(<\mathrm{L}_{b o l}>\sim 8 \times 10^{45} \mathrm{erg} \mathrm{s}^{-1}\right)$ where several theoretical models invoke major galaxy mergers as the main fueling channel for black hole accretion. To derive robust estimates of the host galaxy properties, we use an SED fitting technique to distinguish the AGN and host galaxy emission. We find that at $\mathrm{z} \sim 1, \approx 62 \%$ of Type- 2 QSOs hosts are actively forming stars and that their rates are comparable to those measured for normal star-forming galaxies. The fraction of star-forming hosts increases with redshift: $\approx 71 \%$ at $\mathrm{z} \sim 2$, and $100 \%$ at $\mathrm{z} \sim 3$. We also find that the evolution from $\mathrm{z} \sim 1$ to $\mathrm{z} \sim 3$ of the specific star-formation rate of the Type-2 QSO hosts is in excellent agreement with that measured for star-forming galaxies.
\end{abstract}

Keywords. galaxies: active - quasars: general - X-rays: general

\section{Introduction}

There is strong observational evidence that the formation and growth of supermassive black holes (SMBHs) and their host galaxies are related processes. From the theoretical side, a number of models have been proposed to link the formation and evolution of SMBHs to the structure formation over cosmic time. Some of these semi-analytical models and hydrodynamical simulations (e.g. Springel et al. 2005) invoke major mergers of gas-rich galaxies as the mechanism enabling the fueling of the central SMBHs and the building of the galaxy's bulge. Alternative fueling mechanisms have been discussed in the literature, including minor-mergers (e.g. Johansson et al. 2009), bars (e.g. Jogee 2004), disk instabilities (e.g. Genzel et al. 2008), and recycled gas from dying stars (e.g. Ciotti et al. 2010). Hopkins \& Hernquist 2009 argued that such alternative processes may be enough to fuel Seyfert-like AGNs, but that extreme mechanisms such as major mergers are necessary to provide the gas supply needed for bright quasars. In this framework, the interest in studying the host galaxy properties of bright quasars is clear. We have studied a large sample of X-ray selected Type-2 QSOs from the XMM-COSMOS survey (Cappelluti et al. 2009) to extend to quasar luminosities the study of the host galaxy properties of AGN.

\section{Sample selection and SED fitting}

The final sample includes 142 objects (Mainieri et al. 2011) that satisfy the following criteria: a) de-absorbed $\mathrm{L}_{\mathrm{X}}[0.5-10 \mathrm{keV}]>10^{44} \mathrm{erg} \mathrm{s}^{-1}$; b) $\mathrm{N}_{\mathrm{H}}>10^{22} \mathrm{~cm}^{-2}$. From 
the absorption-corrected luminosities in the rest-frame [2-10 keV] energy band, we estimated the bolometric luminosities for the Type-2 QSOs using the luminosity-dependent bolometric correction factor $\left(k_{2-10 k e V}\right)$ of Marconi et al. 2004. For the X-ray luminosities sampled by our Type-2 QSOs, $k_{2-10 k e V}$ is in the range [25,120], and the bolometric luminosities cover the range $\mathrm{L}_{\mathrm{bol}}=\left[1.2 \times 10^{45}, 4.5 \times 10^{47}\right] \mathrm{erg} \mathrm{s}^{-1}$ with a mean value of $<\mathrm{L}_{\mathrm{bol}}>\approx 8 \times 10^{45} \mathrm{erg} \mathrm{s}^{-1}$. The main goal of our study is to use the rich multiwavelength coverage of the COSMOS field to constrain the properties of the galaxies hosting Type-2 QSOs. The technique we used is a detailed model fitting of the total SED of the Type-2 QSOs for 14 different photometric bands that encompass optical to MIR wavelengths: six SUBARU bands (B, V, g, r, i, z); U, J, and K bands from CFHT; four Spitzer/IRAC channels, and $24 \mu \mathrm{m}$ from Spitzer/MIPS (see Brusa et al. 2010 and references therein). We fitted the observed SED with a grid of models compiled by combining AGN and host galaxy templates. For the AGN component, we adopted the Richards et al. 2006 mean QSO SED derived from the study of 259 IR selected quasars with both Sloan Digital Sky Survey and Spitzer photometry. For the host galaxy, we created a library of synthetic spectra using the well-known stellar population synthesis models of Bruzual \& Charlot 2003. We compiled ten exponentially declining star-formation histories (SFH) $S F R \propto e^{-t_{\text {age }} / \tau}$ with e-folding times $(\tau)$ ranging from 0.1 to $30 \mathrm{Gyr}$, plus a model with constant star formation. For each one of the Type-2 QSOs, we derived the best fit SED from the minimization of the $\chi^{2}$ comparing the observed and template fluxes at the redshift of the source.

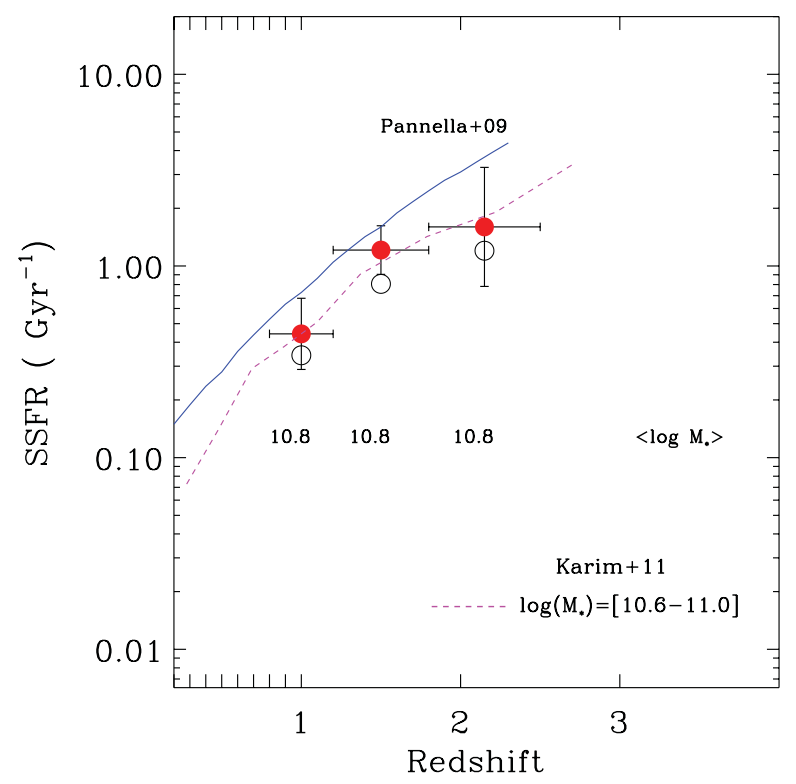

Figure 1. Evolution of the average SSFR for Type-2 QSOs hosts with $\log \left(M_{\star}\right)=[10.6,11.0] \mathrm{M}_{\odot}$ from $\mathrm{z} \sim 1$ to $\mathrm{z} \sim 2$. Empty circles indicate the average SSFR per redshift bin if an unobscured AGN component is included in the SED fitting procedure. The continuum line reproduces the evolution law found by Pannella et al. 2009 for star-forming galaxies up to $\mathrm{z} \sim 2$. The dashed line instead is the best fit to the SSFR redshift evolution derived by Karim et al. 2011 in the mass range $\log \left(\mathrm{M}_{\star}\right)=[10.6,11.0] \mathrm{M}_{\odot}$. 


\section{Host galaxy properties}

Our main aim is to compare the level of star formation in the hosts of the Type2 QSOs with that measured for "non-active" galaxies. Surveys of the local Universe (Brinchmann et al. 2004; Salim et al. 2005) found that galaxies can be divided mainly into two populations: star-forming objects exhibiting a continuous increase in SFR with $\mathrm{M}_{\star}$ along a tight correlation and quenched galaxies with little star formation and high stellar masses. The tight correlation between SFR and $M_{\star}$ for star-forming galaxies has been confirmed to already be in place at higher redshifts (Noeske et al. 2007a, and Elbaz et al. 2007 up to z $\approx 1$; Daddi et al. 2007, and Pannella et al. 2009 up to z $\approx 2$ ), also when using PACS/Herschel observations (Rodighiero et al. 2010). We refer to it as the main sequence (MS) of star-forming galaxies following Noeske et al. 2007a. The slopes of the MS at different redshifts appear to be the same but the normalization has decreased by a factor $\approx 3.7$ going from $\mathrm{z} \approx 2$ to $\mathrm{z} \approx 1$, and an additional factor $\approx 7$ at $\mathrm{z} \approx 0$ (Daddi et al. 2007). This decrease in the activity of star-forming galaxies of a given stellar mass reflects the decline in the global SFR density with time, and a likely cause of this is gas exhaustion (e.g. Noeske et al. 2007b).

In the redshift range $0.8<\mathrm{z}<1.2$, we have 35 Type- 2 QSOs. We find that $\approx 62 \%$ of the Type-2 QSO host in this redshift range are actively forming stars. We repeated a similar analysis for the hosts in the three higher redshifts bins: $1.2<z<1.8$ (36 objects), $1.8<z<2.5$ (28 objects), and $2.5<z<3.2$ (12 objects). First, we find that the fraction of star-forming galaxies between our Type-2 QSO hosts increases with redshift: $66 \%, 71 \%$, and $100 \%$ respectively. We then looked at the evolution of the SSFR for Type-2 QSO hosts between redshift $z=0.8$ and $z=3$ as shown in Fig. 1 . We find that the redshift evolution of the specific star formation rate for the hosts of Type-2 QSOs is in excellent agreement with that observed for star-forming galaxies in the redshift range $1<z<3$ : the average SSFR increase by a factor $\approx 3.6$ going from $\mathrm{z} \sim 1$ to $\mathrm{z} \sim 2$, and remains constant at higher redshifts.

\section{References}

Brinchmann, J., Charlot, S., White, S. D. M., et al. 2004, MNRAS, 351, 1151

Brusa, M., Civano, F., Comastri, A. et al. 2010, ApJ, 716, 348

Bruzual, G. \& Charlot, S., 2003, MNRAS, 344, 1000

Cappelluti, N., Brusa, M., Hasinger, G. et al. 2009, A\&A A, 497, 635

Ciotti, L., Ostriker, J. P., \& Proga, D. 2010, ApJ, 717, 708

Daddi, E., Dickinson, M., Morrison, G. et al. 2007, ApJ, 670, 156

Elbaz, D., Daddi, E., Le Borgne, D. et al., 2007, A\& $A, 468,33$

Genzel, R., Burkert, A., Bouche, N. et al., 2008, ApJ, 687, 59

Hopkins, P. F. \& Hernquist, L. 2009, ApJ, 694, 599

Johansson, P. H., Naab, T., \& Burkert, A. 2009, ApJ, 690, 802

Jogee, S. 2004, Physics of Active Galactic Nuclei at all Scales, 693, 143

Karim, A., Schinnerer, E., Martinez-Sansigre, A. et al., 2011, ApJ, 730, 61

Mainieri, V., Bongiorno, A., Merloni, A. et al., 2011, A\& $A, 535,80$

Marconi, A., Risaliti, G., Gilli, R. et al. 2004, MNRAS, 351, 169

Noeske, K. G., Weiner, B. J., Faber, S. M. et al. 2007a, ApJ, 660, L43

Noeske, K. G., Faber, S. M., Weiner, B. J. et al. 2007b, ApJ, 660, L47

Pannella, M., Carilli, C. L., Daddi, E. et al. 2009, ApJ, 698, L116

Richards, G. T., Lacy, M., Storrie-Lombardi, L. J. et al. 2006, ApJS, 166, 470

Rodighiero, G., Cimatti, A., Gruppioni, C. et al. 2010, A\&\&A, 518, L25

Salim, S., Charlot, S., Rich, R. M. et al. 2005, ApJ, 619, L39

Springel, V., Di Matteo, T., \& Hernquist, L. 2005, MNRAS, 361, 776 